\title{
Cognitive Impairment in Persons With Rheumatoid Arthritis
}

\author{
So Young Shin, PhD, RN, GCNS-BC, Patricia Katz, PhD, Margaret Wallhagen, PhD, GNP- \\ BC, and Laura Julian, PhD \\ University of California, San Francisco
}

\begin{abstract}
Objective-To explore the prevalence and possible predictors of cognitive impairment in persons with rheumatoid arthritis (RA).

Methods-Individuals from a longitudinal cohort study of RA participated in a study visit that included a range of physical, psychosocial, and biologic metrics. Cognitive function was assessed using a battery of 12 standardized neuropsychological measures yielding 16 indices. Subjects were classified as "impaired" if they performed 1 SD below age-based population norms on at least 4 of 16 indices. Logistic regression analyses were conducted to identify which of the following were significant predictors of cognitive impairment: sex, race, income, education, depression, disease duration, disease severity, C-reactive protein (CRP) level, glucocorticoid use, and cardiovascular disease (CVD) risk factors.
\end{abstract}

Results-A total of 115 subjects with a mean \pm SD age of $58.6 \pm 10.8$ years were included; $64 \%$ were women and $81 \%$ were white. The proportion of persons who were classified as cognitively impaired was $31 \%$. Education, income, glucocorticoid use, and CVD risk factors independently predicted cognitive impairment, controlling for sex, race, disease duration, disease severity, CRP level, and depression. Individuals with cognitive impairment were more likely to have low education (odds ratio [OR] 6.18, 95\% confidence interval [95\% CI] 1.6 -23.87), have low income (OR 7.12, 95\% CI 1.35-37.51), use oral glucocorticoids (OR 2.92, 95\% CI 1.05-8.12), and have increased CVD risk factors (OR 1.61, 95\% CI 1.19-2.17 per risk factor).

Conclusion-The findings of this study suggest that the burden of cognitive impairment in RA is significant, and future studies identifying specific etiologic contributors to cognitive impairment are warranted.

\section{INTRODUCTION}

Studies in the general population have found cognitively impaired persons to have increased functional difficulties and reduced well-being (1). For persons with chronic diseases such as rheumatoid arthritis (RA), intact cognitive function is critical for the successful performance of daily activities, managing and adhering to treatment regimens, and planning and initiating activities based on one's current health condition (2). Mechanisms that have been linked to cognitive impairment in the general population, such as systemic inflammation (3) and

\footnotetext{
Address correspondence to So Young Shin, PhD, RN, GCNS-BC, Department of Physiological Nursing, University of California, San Francisco, 2 Koret Way, N611M, San Francisco, CA 94143-0602. soyoung.shin@ucsf.edu.

AUTHOR CONTRIBUTIONS

All authors were involved in drafting the article or revising it critically for important intellectual content, and all authors approved the final version to be submitted for publication. Dr. Shin had full access to all of the data in the study and takes responsibility for the integrity of the data and the accuracy of the data analysis.

Study conception and design. Shin, Katz, Wallhagen, Julian.

Acquisition of data. Katz.

Analysis and interpretation of data. Shin, Katz, Wallhagen, Julian.
} 
cardiovascular disease (CVD) (4), have particular relevance for RA. Yet, very little is known about potential factors that contribute to decreased cognitive function in persons with RA.

Two primary studies have evaluated cognitive dysfunction in well-characterized cohorts of RA patients using a comprehensive neuropsychological test battery that extends beyond bedside mental status screening examinations. Bartolini et al (5) observed that cognitive dysfunction was common in RA patients, with prevalence rates ranging from 38\% (divided/ sustained attention and mental flexibility) to $71 \%$ (visuo-spatial and planning functions). In this cohort, cognitive dysfunction was also associated with neuroimaging findings, including hypoperfusion on brain single-photon-emission computed tomography and increased white matter alterations on magnetic resonance imaging. Additionally, Appenzeller et al (6) found cognitive impairment in $30 \%$ of the RA cohort as compared to $8 \%$ of healthy controls. These few studies have important implications in that they highlight the potential burden of cognitive impairment and its possible risk factors in persons with RA.

Because RA is a chronic incurable disease, persons with RA may have the increased burden of both age-related and disease-related cognitive decline as they age. A number of studies have assessed cognitive dysfunction as one of the risk factors that might exacerbate functional difficulties and well-being in individuals with various chronic health conditions (7-9). Assessing a wide spectrum of cognitive domains in RA patients may help in understanding the burden of cognitive problems in these patients and developing targeted interventions to minimize adverse outcomes on health status. Therefore, the purpose of this study was to explore the prevalence of cognitive impairment in a cohort of individuals with RA, and to identify the specific factors that are associated with cognitive impairment in these persons. The hypothesis was that disease-related factors would significantly affect cognitive impairment in persons with RA after controlling for sociodemographic variables.

\section{SUBJECTS AND METHODS}

\section{Sample and setting}

Subjects were drawn from the University of California, San Francisco (UCSF) RA Panel, which was initiated in 1982. Details about enrollment and data collection have been described previously (10). Trained interviewers have conducted structured annual telephone interviews that included questions on sociodemographic characteristics, general health status, disease-related symptoms, medication use, psychological health status, physical function, and disability.

At the end of the telephone interviews in study years 2007-2009, participants who lived in the San Francisco Bay area and were willing to travel to the UCSF were recruited for inperson assessments at the UCSF Clinical and Translational Science Institute Clinical Research Services (CRS) facility. In 2009, an additional 44 subjects were recruited from the UCSF rheumatology clinic and from individuals who had participated in another study of RA and had agreed to be contacted for other studies. In total, 144 individuals (60\% of those who were recruited and were eligible) participated in the CRS visits.

The CRS visits included a range of physical, psychosocial, cognitive, and biologic measures. Data from the CRS visits were merged with data collected during the standardized telephone interviews. Finally, 115 subjects who had complete data on all outcomes and covariates of interest were included in this study; of the participants who were excluded from the analyses, the majority were missing data on family income $(n=9)$ and neuropsychological performance $(\mathrm{n}=9), 7$ were missing data on CVD risk factors, and 6 were missing data on 
disease severity. The research protocol was approved by the UCSF Committee on Human Research, and all subjects gave their informed consent to participate.

\section{Measures}

Cognitive function-Cognitive function was assessed using a standardized neuropsychological battery that was modified from the American College of Rheumatology neuropsychological battery (11). This battery is primarily designed for use in a comparable rheumatic condition, systemic lupus erythematosus (SLE), and has been deemed reliable and valid $(12,13)$. We modified it for use in RA to minimize or control for the effects of handmotor dysfunction.

Neuropsychological tests included the California Verbal Learning Test-II (14) Learning, Short Delay, and Long Delay Recall; the Rey-Osterrieth Complex Figure Test (15) Copy Trial, Immediate Delay, and Long Delay Recall; the Controlled Oral Word Association Test and the Animal Naming Test (16); the oral version of the Symbol Digit Modalities Test (17); the Delis Kaplan Executive Function Scale, including Card Sorting Test (total correct), Design Fluency Test (total correct), Trail Making Test (timing for sequencing/shifting condition), and Color Word Inference Test (18) Inhibition and Switching Conditions; the Wechsler Adult Intelligence Scale-III Digit Span Backwards Test (19); and the short form Judgment of Line Orientation Test $(20,21)$. The duration of the neuropsychological battery was approximately $60-80$ minutes.

Neuropsychological tests were scored to yield Z scores based on age-stratified population norms, and 16 neuropsychological indices were derived. Using conventional cut points, subjects were classified as "impaired" if they performed 1 SD below age-stratified population norms for each cognitive test $(12,22)$. A total cognitive function score was calculated by summing the number of tests on which individuals were classified as "impaired," ranging from $0-16$ (higher scores indicating greater impairment). For subjects who completed at least $80 \%$ of the 16 subtests ( $\geq 13$ ), but did not complete $1-3$ of the subtests, the mean $\mathrm{Z}$ score of the subtests that they did complete was substituted as the scores for the missing subtests before the total cognitive function score was created. Finally, persons with total cognitive function scores of $\geq 4$ were classified as "cognitively impaired."

Covariates-Sociodemographics and disease characteristics (i.e., age, sex, race, educational level, marital status, family income, presence of hypertension, use of medications including antihypertensive medicines and oral glucocorticoids, smoking status, disease duration, disease severity, and depression) were assessed based on self-reported information. Blood samples for measurement of high-sensitivity C-reactive protein (CRP) level, total cholesterol, and high-density lipoprotein (HDL) cholesterol were collected during the CRS visit and sent to a commercial laboratory for analysis. Blood pressure, as well as height and weight to calculate body mass index (BMI), were also measured during the study visit.

Depression was assessed using the Mini International Neuropsychiatric Interview (MINI) (23-25), a short diagnostic structured interview corresponding to the Diagnostic and Statistical Manual of Mental Disorders, Fourth Edition criteria for the axis I psychiatric disorders. The MINI was administered by study clinical evaluators trained and supervised by a clinical psychologist (LJ). The MINI has been deemed reliable and valid across many populations $(23,24)$.

Severity of RA was assessed using the Rheumatoid Arthritis Disease Activity Index (RADAI) $(26,27)$, a patient-assessed measure of disease activity in RA covering global disease activity in the past 6 months (current joint pain, tenderness, and swelling, and 
current duration of morning stiffness). RADAI scores range from $0-10$, with higher scores reflecting greater disease activity. It has been shown to be reliable and valid $(26,27)$.

CVD risk factor scores were generated based on variables in the CVD risk score profiles from the Framingham Heart Study $(28,29)$. Scores were calculated as the total number of the following CVD risk factors that were present: hypertension, systolic blood pressure $>140$ $\mathrm{mm} \mathrm{Hg}$, antihypertensive medication use, total cholesterol $>200 \mathrm{mg} / \mathrm{dl}$, HDL cholesterol $<60 \mathrm{mg} / \mathrm{dl}$, current smoking, and obesity (BMI $>30 \mathrm{~kg} / \mathrm{m}^{2}$ ). CVD risk factor scores ranged from $0-7$, with higher scores indicating greater CVD risk.

\section{Statistical analyses}

Chi-square analyses and $t$-tests were used to determine whether significant differences existed between the cognitively impaired and unimpaired groups. Logistic regression analyses were used to identify potential predictors of cognitive impairment and included variables that have been linked with cognitive impairment in previous studies among individuals with chronic health conditions. Sex, race, education, income, duration of RA, severity of RA, depression, CRP level, oral glucocorticoid use, and CVD risk factor scores were assessed as the potential predictors of cognitive impairment. The limit for significance was set at 2-tailed $\alpha=0.05$. All analyses were conducted using SPSS, version 19.0.

\section{RESULTS}

Subject characteristics are presented in Table 1. Mean \pm SD total cognitive function score was $2.5 \pm 2.2$ and ranged from $0-10$. The proportion of persons who were classified as cognitively impaired on each test ranged from $8 \%$ (semantic fluency test) to 29\% (visuospatial learning/memory test). The proportion of persons classified as cognitively impaired ( $\geq 4$ of 16 subtests) was $31 \%$ (Figure 1). There were no significant differences between cognitively impaired and unimpaired groups except for 3 variables. Cognitively impaired persons were more likely to have $<12$ years of education $(P=0.032),<\$ 20,000$ of income $(P$ $=0.045)$, and more CVD risk factors $(P=0.003)$ than unimpaired persons.

Sex, race, education, income, depression, duration of RA, severity of RA, CRP level, oral glucocorticoid use, and the number of CVD risk factors were included in multivariate logistic regression models (Table 2). These 10 predictors explained $24-34 \%$ of the variance in cognitive impairment. Of the clinical variables, current oral glucocorticoid use and CVD risk factor score independently predicted cognitive impairment $\left(\chi^{2}[10]=31.60, P<0.005\right)$; education and income were the only demographic factors associated with cognitive impairment (outcome was already adjusted for age) even after controlling for other covariates. Individuals with cognitive impairment were more likely to report current use of oral glucocorticoids (odds ratio [OR] 2.92, 95\% confidence interval [95\% CI] 1.05-8.12) and have a greater number of CVD risk factors (OR 1.61, 95\% CI 1.19-2.17 per risk factor), controlling for all other variables in the model. Low education (OR 6.18, 95\% CI 1.6023.87) and low income (OR 7.12, 95\% CI 1.35-37.51) were also significantly associated with cognitive impairment, whereas sex, race, disease duration, disease severity, CRP level, and depression were not significant predictors of cognitive impairment in this cohort.

\section{DISCUSSION}

In this study, we sought to explore the prevalence and clinical predictors of cognitive impairment in persons with RA. The proportion of persons who were classified as cognitively impaired on each test ranged from 8-29\%. Approximately one-third of subjects were classified as cognitively impaired on $\geq 4$ tests. In multivariate models, adjusting for relevant sociodemographics, only oral glucocorticoid use and cumulative number of CVD 
risk factors emerged as the clinical factors independently associated with the presence of cognitive impairment.

More than $20 \%$ of subjects were found to be cognitively impaired in domains evaluating executive function (28\% on the Design Fluency Test and $21 \%$ on the Trail Making Test). In addition, $29 \%$ and $18 \%$ of subjects were classified as cognitively impaired in domains evaluating visuospatial learning/memory and verbal learning/memory, respectively. These results are analogous to previous studies. For example, Appenzeller et al (6) found cognitive impairment in $30 \%$ of the RA cohort, with worse outcomes in domains evaluating verbal fluency and episodic memory. We found slightly lower prevalence rates in comparison to another study by Bartolini et al (5), who observed cognitive dysfunction in $38-71 \%$ of their cohort of RA patients. Although direct comparisons among studies may not be possible due to different classifications of cognitive impairment and diverse assessment methods used, these results do imply the significance of cognitive problems in RA. Further studies that assess cognitive function in RA with standardized criteria and methodologies are needed.

Lower levels of socioeconomic status (e.g., education and income) are generally well-known risk factors of poorer performance on cognitive tasks in individuals with or without multiple chronic problems (30-33). Comparable to those studies of cognitive function in chronic disease, both lower education and lower income were independent predictors of cognitive impairment in persons with RA. The findings of oral glucocorticoid use and CVD risk factors emerging as significant predictors of cognitive impairment in RA patients is notable. In this study, most subjects were taking relatively low-dose glucocorticoids; mean \pm SD daily glucocorticoid dosage was $2.1 \pm 4.7 \mathrm{mg}$, and only 14 individuals were taking more than $5 \mathrm{mg}$ of glucocorticoids per day. Nevertheless, subjects with any oral glucocorticoid use were approximately 3 times more likely to be cognitively impaired than those without oral glucocorticoid use. This finding is consistent with previous research. Excessive circulatory levels of corticosteroids were observed to be associated with cognitive impairment in various disease states (34). Wolkowitz et al (35) found that even a single dose ( $1 \mathrm{mg}$ dose of dexamethasone) or short-term use of corticosteroids (80 $\mathrm{mg}$ dose of prednisone for 5 days) was significantly related to memory problems and raised the possible adverse effect of corticosteroids on cognitive function. This result implies that even patients with relatively low-dose glucocorticoid use may be at risk for cognitive problems.

Subjects with a greater number of CVD risk factors were more likely to be cognitively impaired in this study. Cardiovascular-related risk factors are known predictors of cognitive decline $(4,36,37)$, and executive function may be particularly vulnerable to the effect of CVD (38). RA patients are more likely to have cardiovascular-related morbidity/mortality compared to non-RA patients $(39,40)$. Therefore, comorbid CVD risk factors including hypertension, hyperlipidemia, obesity, or current smoking may increase the prevalence of cognitive impairment in RA. In addition, the influence of glucocorticoid use and CVD may be interconnected, with some studies suggesting that glucocorticoid use may also confer a direct risk for CVD in rheumatic disease $(41,42)$. Previous research has reported that RA patients with long-term low- or middle-dose glucocorticoid use had a higher prevalence of some aspects of CVD risk and incidence including hypertension and myocardial infarction than those with no glucocorticoid use $(41,42)$. Mazzantini et al $(41)$ found that medium-dose long-term steroid users had a higher prevalence of hypertension compared to no or limited steroid users. Additionally, Davis et al (43) found that rheumatoid factor-positive patients with RA had an increased risk of CVD events after using glucocorticoids. The increased risk of cardiovascular conditions may interact with long-term glucocorticoid use to influence cognitive function. Patients with RA may be cognitively impaired not by the direct impact of disease process or symptoms such as duration, severity, or inflammation, but by the indirect 
impact of comorbid CVD risk factors or even low-dose glucocorticoid use, a common regimen of RA management.

A few factors that are related to RA activity or severity have been shown to be also associated with cognitive dysfunction in previous studies. Inflammatory markers such as CRP level or interleukin- 6 are observed to be associated with incident cognitive impairment and cognitive decline in the general population $(3,44)$ and in other rheumatic conditions including SLE (45). Disease symptoms, such as chronic pain and psychological distress, have been linked to cognitive impairment (46). However, these factors did not emerge as significant predictors of cognitive impairment in this study. Depression is commonly observed as a risk factor for cognitive decline in other populations (47), but did not emerge as a significant predictor in this study, perhaps due to the relatively low prevalence of major depressive disorder in this cohort or perhaps due to the use of a dichotomous diagnostic assessment of depression in lieu of a severity scale of depressive symptomatology. More studies are needed to identify the exact contributions of these factors to cognitive dysfunction in RA patients.

This study has some limitations that should be mentioned. The sample for this study may not be representative of all patients with RA for several reasons. Many subjects were participants of a long-term prospective study of RA (active since 1983) and may be relatively healthy survivors who have been able to participate in long-term research studies. Only persons who lived in the San Francisco Bay area and were able to travel to the UCSF clinical research center were included in the study, perhaps also biasing the sample toward more healthy individuals. Subjects were primarily white with relatively high education and income, which might limit the generalization of the study findings to certain groups.

The classification criteria of cognitive impairment (i.e., if subjects performed 1 SD below age-adjusted population norms on $25 \%$ of the battery) in this study may be less conservative than some other studies, but also are comparable in stringency to other studies in rheumatic disease $(12,22)$. It was our intent to evaluate the spectrum of cognitive impairment in this condition, as even mild levels of impairment can disrupt daily functioning. Additionally, this cohort was highly educated and we wanted to minimize risk of false-negatives in our criteria selection.

Individuals who are cognitively impaired may be inaccurate reporters of RA disease symptoms. Information on long-term or cumulative glucocorticoid use was not available for this cohort, but would have provided added information regarding the role of glucocorticoids and cognitive impairment in RA. Other factors that could potentially account for cognitive impairment, e.g., sleep quality, were not assessed in this study. This was a cross-sectional study, and therefore cannot provide causal information among the variables. In spite of statistically significant findings regarding the relationship between the variables of interest, the causal pathway to cognitive impairment could not be determined in this cross-sectional study. A longitudinal study design is required to identify the causal relationship between the variables of interest.

In spite of some limitations, this study has strengths and important implications. This is one of few studies that assessed a wide spectrum of cognitive domains in RA patients using a range of neuropsychological tests. We observed that a substantial subset of our participants met criteria for cognitive impairment in a range of cognitive domains including visuo-spatial functioning and executive functioning. Individuals with impairments in these domains may have difficulties in performing daily activities and maintaining self-management regimens. Additionally, many interventions for RA often require changes in knowledge, behavior, and lifestyle, which likely require intact functioning in these domains. In particular, poor 
executive function may aggravate poor physical, psychological, and social health in persons with RA (2). This study provides support for the use of a comprehensive cognitive evaluation beyond traditional bedside screening measures as they may be insufficient and inappropriate in assessing this complex cognitive subdomain.

This study has significant implications for clinical practice. As mentioned previously, intact cognitive function in patients with chronic diseases is important for performing fundamental daily activities and managing complex health conditions such as RA. Identifying factors that affect cognitive impairment in persons with RA is an initial step for developing effective and targeted interventions that minimize its adverse outcomes. The findings of this study enhance our understanding of cognitive impairment in RA and will help lay the foundation for designing targeted interventions to prevent and improve cognitive function in these persons. Additionally, persons with RA and cognitive impairment may benefit from interventions modified for cognitively impaired patients or designed to improve cognitive function.

The results of this study emphasize the burden of cognitive impairment in RA and the importance of cognitive function assessment in clinical settings as a significant factor in RA management. Health care providers should cautiously assess cognitive status of RA patients, especially those with oral glucocorticoid use and with CVD risk factors. Substantial attention in recent years has been paid to the burden of CVD in RA. It is well known in the general population that CVD conveys risk for the development of cognitive dysfunction and management and prevention of CVD may improve cognitive function or delay the onset of cognitive decline. To date, this is the first study to evaluate the role of CVD risk in relation to cognitive function in RA. Future studies investigating both the role of CVD in precipitating cognitive alterations and the treatment and prevention of CVD in alleviating these neuropsychiatric manifestations are warranted.

In conclusion, intact cognitive function is critical for maintaining functional independence and well-being in persons with chronic diseases. Almost one-third of RA patients were found to be cognitively impaired in this study. Persons with less education, less income, oral glucocorticoid use, and increased CVD risk factors were more likely to be cognitively impaired. The classic features of RA (e.g., disease duration or severity) were not associated with cognitive impairment in persons with RA. However, other treatment-related factors (e.g., glucocorticoid use) and systemic features of RA that are increasingly recognized as a significant consequence of RA disease process (e.g., CVD risk) were significantly associated with cognitive impairment in this population. The findings of this study suggest that the burden of cognitive impairment in RA may be significant, and future studies identifying specific etiologic contributors to cognitive impairment are warranted.

\section{Acknowledgments}

Supported by the NIH/National Institute of Arthritis and Musculoskeletal and Skin Diseases (grant P60AR-053308) and the National Center for Research Resources, Clinical and Translational Science Institute, University of California, San Francisco (grant UL-1RR-024131).

\section{REFERENCES}

1. Bennett D, Wilson R, Schneider J, Evans D, Beckett L, Aggarwal N, et al. Natural history of mild cognitive impairment in older persons. Neurology. 2002; 59:198-205. [PubMed: 12136057]

2. Abeare CA, Cohen JL, Axelrod BN, Leisen JC, Mosley-Williams A, Lumley MA. Pain, executive functioning, and affect in patients with rheumatoid arthritis. Clin J Pain. 2010; 26:683-689. [PubMed: 20862788] 
3. Gimeno D, Marmot MG, Singh-Manoux A. Inflammatory markers and cognitive function in middle-aged adults: the Whitehall II study. Psychoneuroendocrinology. 2008; 33:1322-1334. [PubMed: 18774232]

4. Meyer JS, Rauch GM, Rauch RA, Haque A, Crawford K. Cardiovascular and other risk factors for Alzheimer's disease and vascular dementia. Ann N Y Acad Sci. 2000; 903:411-423. [PubMed: 10818532]

5. Bartolini M, Candela M, Brugni M, Catena L, Mari F, Pomponio G, et al. Are behaviour and motor performances of rheumatoid arthritis patients influenced by subclinical cognitive impairments? A clinical and neuroimaging study. Clin Exp Rheumatol. 2002; 20:491-497. [PubMed: 12175104]

6. Appenzeller S, Bertolo MB, Costallat LT. Cognitive impairment in rheumatoid arthritis. Methods Find Exp Clin Pharmacol. 2004; 26:339-343. [PubMed: 15319812]

7. Auyeung T, Kwok T, Lee J, Leung P, Leung J, Woo J. Functional decline in cognitive impairment: the relationship between physical and cognitive function. Neuroepidemiology. 2008; 31:167-173. [PubMed: 18784415]

8. Dunlop DD, Semanik P, Song J, Manheim LM, Shih V, Chang RW. Risk factors for functional decline in older adults with arthritis. Arthritis Rheum. 2005; 52:1274-1282. [PubMed: 15818691]

9. Raji MA, Kuo YF, Snih SA, Markides KS, Peek MK, Ottenbacher KJ. Cognitive status, muscle strength, and subsequent disability in older Mexican Americans. J Am Geriatr Soc. 2005; 53:14621468. [PubMed: 16137273]

10. Katz PP, Morris A, Yelin EH. Prevalence and predictors of disability in valued life activities among individuals with rheumatoid arthritis. Ann Rheum Dis. 2006; 65:763-769. [PubMed: 16249225]

11. ACR Ad Hoc Committee on Neuropsychiatric Lupus Nomenclature. The American College of Rheumatology nomenclature and case definitions for neuropsychiatric lupus syndromes. Arthritis Rheum. 1999; 42:599-608. [PubMed: 10211873]

12. Kozora E, Ellison M, West S. Reliability and validity of the proposed American College of Rheumatology neuropsychological battery for systemic lupus erythematosus. Arthritis Rheum. 2004; 51:810-818. [PubMed: 15478145]

13. Kozora E, Arciniegas DB, Zhang L, West S. Neuropsychological patterns in systemic lupus erythematosus patients with depression. Arthritis Res Ther. 2007; 9:R48. [PubMed: 17504538]

14. Delis, D.; Kramer, J.; Kaplan, E.; Ober, B. California verbal learning test: adult version. San Antonio (TX): Psychological Corporation; 1987.

15. Rey A, Osterrieth P. Translations of excerpts from Andre Rey's psychological examination of traumatic encephalopathy and P. A. Osterrieth's “The Complex Figure Copy Test.”. Clin Neuropsychologist. 1993; 7:3-15.

16. Borkowski J, Benton A, Spreen O. Word fluency and brain damage. Neuropsychologia. 1967; 5:135-140.

17. Smith, A. Symbol digit modalities test: manual. Rev. Los Angeles: Western Psychological Services; 1982. 1982.

18. Delis, D. Delis-Kaplan Executive Function Scale. San Antonio (TX): Psychological Corporation; 2001.

19. Wechsler, D. Wechsler Adult Intelligence Scale-III (WAIS-III). San Antonio (TX): Psychological Corporation; 1997.

20. Woodard JL, Benedict RH, Roberts VJ, Goldstein FC, Kinner KM, Capruso DX, et al. Short-form alternatives to the Judgment of Line Orientation Test. J Clin Exp Neuropsychol. 1996; 18:898904. [PubMed: 9157113]

21. Benton, AL. Contributions to neuropsychological assessment: a clinical manual. New York: Oxford University Press; 1994.

22. Kozora E, Thompson L, West S, Kotzin B. Analysis of cognitive and psychological deficits in systemic lupus erythematosus patients without overt central nervous system disease. Arthritis Rheum. 1996; 39:2035-2045. [PubMed: 8961909]

23. Lecrubier Y, Sheehan D, Weiller E, Amorim P, Bonora I, Harnett Sheehan K, et al. The Mini International Neuropsychiatric Interview (MINI): a short diagnostic structured interview. Reliability and validity according to the CIDI. Eur Psychiatry. 1997; 12:224-231. 
24. Sheehan D, Lecrubier Y, Harnett Sheehan K, Janavs J, Weiller E, Keskiner A, et al. The validity of the Mini International Neuropsychiatric Interview (MINI) according to the SCID-P and its reliability. Eur Psychiatry. 1997; 12:232-241.

25. Sheehan DV, Lecrubier Y, Sheehan KH, Amorim P, Janavs J, Weiller E, et al. The MINIInternational Neuropsychiatric Interview (M.I.N.I.): the development and validation of a structured diagnostic psychiatric interview for DSM-IV and ICD-10. J Clin Psychiatry. 1998; 59(Suppl 20): 22-33. [PubMed: 9881538]

26. Stucki G, Liang M, Stucki S, Bruhlmann P, Michel B. A self-administered rheumatoid arthritis disease activity index (RADAI) for epidemiologic research: psychometric properties and correlation with parameters of disease activity. Arthritis Rheum. 1995; 38:795-798. [PubMed: 7779122]

27. Fransen J, Langenegger T, Michel B, Stucki G. Feasibility and validity of the RADAI, a selfadministered rheumatoid arthritis disease activity index. Rheumatology (Oxford). 2000; 39:321327. [PubMed: 10788543]

28. D’Agostino RB Sr, Vasan RS, Pencina MJ, Wolf PA, Cobain M, Massaro JM, et al. General cardiovascular risk profile for use in primary care: the Framingham Heart Study. Circulation. 2008; 117:743-753. [PubMed: 18212285]

29. Pencina MJ, D’Agostino RB, Larson MG, Massaro JM, Vasan RS. Predicting the 30-year risk of cardiovascular disease. Circulation. 2009; 119:3078-3084. [PubMed: 19506114]

30. Lynch JW, Kaplan GA, Shema SJ. Cumulative impact of sustained economic hardship on physical, cognitive, psychological, and social functioning. N Engl J Med. 1997; 337:1889-1895. [PubMed: 9407157]

31. Adler NE, Ostrove JM. Socioeconomic status and health: what we know and what we don't. Ann N Y Acad Sci. 1999; 896:3-15. [PubMed: 10681884]

32. Lee S, Kawachi I, Berkman LF, Grodstein F. Education, other socioeconomic indicators, and cognitive function. Am J Epidemiol. 2003; 157:712-720. [PubMed: 12697575]

33. Cagney KA, Lauderdale DS. Education, wealth, and cognitive function in later life. J Gerontol B Psychol Sci Soc Sci. 2002; 57:P163-P172. [PubMed: 11867664]

34. Belanoff JK, Gross K, Yager A, Schatzberg AF. Corticosteroids and cognition. J Psychiatr Res. 2001; 35:127-145. [PubMed: 11461709]

35. Wolkowitz OM, Reus VI, Weingartner H, Thompson K, Breier A, Doran A, et al. Cognitive effects of corticosteroids. Am J Psychiatry. 1990; 147:1297-1303. [PubMed: 2399996]

36. Knopman D, Boland L, Mosley T, Howard G, Liao D, Szklo M, et al. Cardiovascular risk factors and cognitive decline in middle-aged adults. Neurology. 2001; 56:42-48. [PubMed: 11148234]

37. Singh-Manoux A, Sabia S, Lajnef M, Ferrie JE, Nabi H, Britton AR, et al. History of coronary heart disease and cognitive performance in midlife: the Whitehall II study. Eur Heart J. 2008; 29:2100-2107. [PubMed: 18648106]

38. Chui H. Dementia due to subcortical ischemic vascular disease. Clin Cornerstone. 2001; 3:40-51. [PubMed: 11432121]

39. Maradit Kremers H, Crowson CS, Nicola PJ, Ballman KV, Roger VL, Jacobsen SJ, et al. Increased unrecognized coronary heart disease and sudden deaths in rheumatoid arthritis: a population-based cohort study. Arthritis Rheum. 2005; 52:402-411. [PubMed: 15693010]

40. Roifman I, Beck PL, Anderson TJ, Eisenberg MJ, Genest J. Chronic inflammatory diseases and cardiovascular risk: a systematic review. Can J Cardiol. 2011; 27:174-182. [PubMed: 21459266]

41. Mazzantini M, Talarico R, Doveri M, Consensi A, Cazzato M, Bazzichi L, et al. Incident comorbidity among patients with rheumatoid arthritis treated or not with low-dose glucocorticoids: a retrospective study. J Rheumatol. 2010; 37:2232-2236. [PubMed: 20843913]

42. Panoulas VF, Douglas KM, Stavropoulos-Kalinoglou A, Metsios GS, Nightingale P, Kita M, et al. Long-term exposure to medium-dose glucocorticoid therapy associates with hypertension in patients with rheumatoid arthritis. Rheumatology (Oxford). 2008; 47:72-75. [PubMed: 18077493]

43. Davis JM III, Maradit Kremers H, Crowson CS, Nicola PJ, Ballman KV, Therneau TM, et al. Glucocorticoids and cardiovascular events in rheumatoid arthritis: a population-based cohort study. Arthritis Rheum. 2007; 56:820-830. [PubMed: 17330254] 
44. Yaffe K, Lindquist K, Penninx B, Simonsick E, Pahor M, Kritchevsky S, et al. Inflammatory markers and cognition in well-functioning African-American and white elders. Neurology. 2003; 61:76-80. [PubMed: 12847160]

45. Shucard JL, Gaines JJ, Ambrus J Jr, Shucard DW. C-reactive protein and cognitive deficits in systemic lupus erythematosus. Cogn Behav Neurol. 2007; 20:31-37. [PubMed: 17356342]

46. Hart RP, Wade JB, Martelli MF. Cognitive impairment in patients with chronic pain: the significance of stress. Curr Pain Headache Rep. 2003; 7:116-126. [PubMed: 12628053]

47. Chodosh J, Kado DM, Seeman TE, Karlamangla AS. Depressive symptoms as a predictor of cognitive decline: MacArthur studies of successful aging. Am J Geriatr Psychiatry. 2007; 15:406415. [PubMed: 17353297] 


\section{Significance \& Innovations}

- For persons with chronic diseases such as rheumatoid arthritis (RA), intact cognitive function is critical for performing daily activities and maintaining disease management skills.

- This is one of the few studies that assessed a wide spectrum of cognitive domains in RA patients using a range of neuropsychological tests.

- The results of this study emphasize the burden of cognitive impairment in RA and the importance of cognitive function assessment in clinical settings as a significant factor in RA management. 


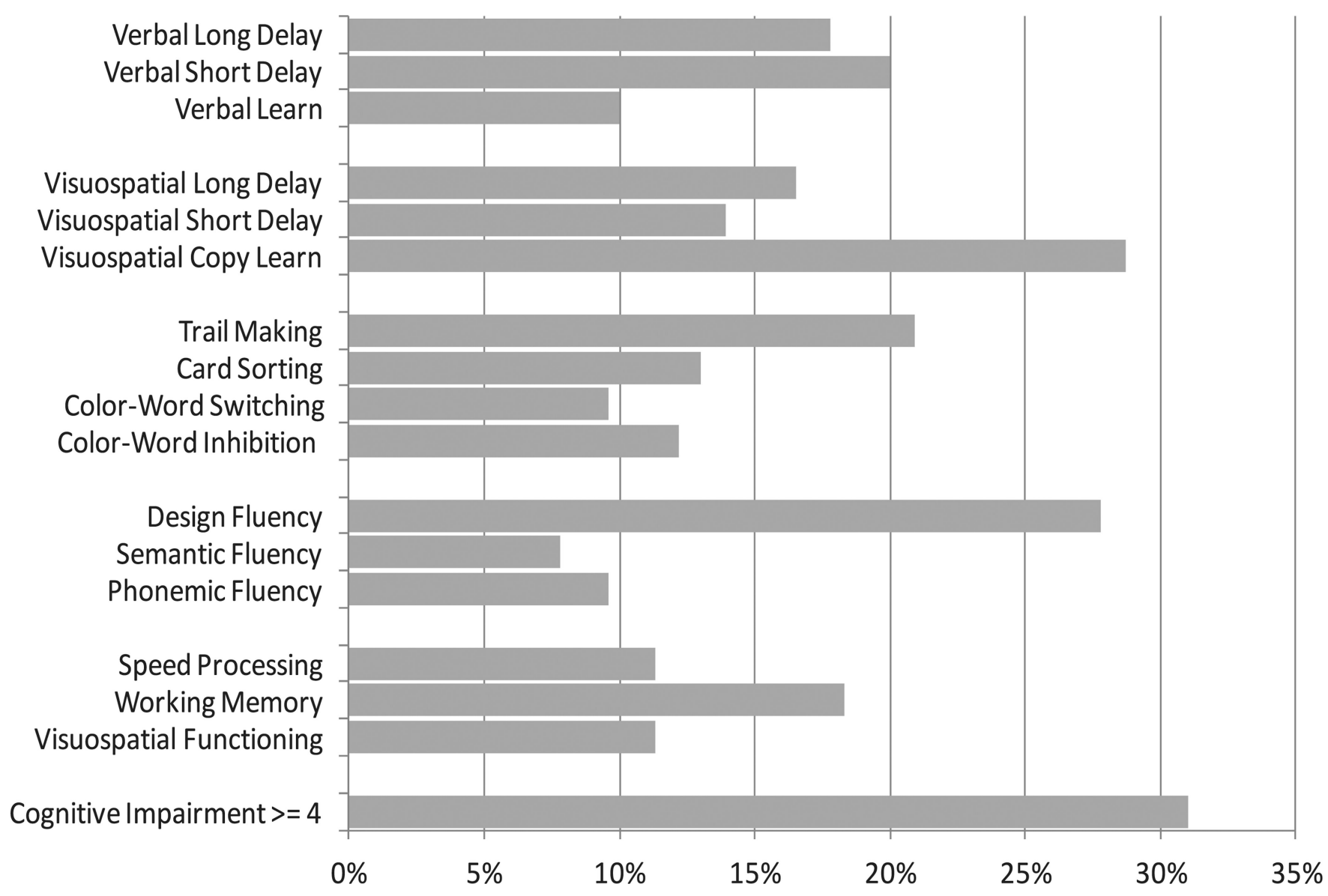

Figure 1.

Characteristics of neuropsychological test performance (percent impaired). 
Table 1

Characteristics of subjects $(\mathrm{n}=115)^{*}$

\begin{tabular}{|lcccc|}
\hline & $\begin{array}{c}\text { Total } \\
(\mathbf{n}=\mathbf{1 1 5})\end{array}$ & $\begin{array}{c}\text { Impaired } \\
(\mathbf{n}=\mathbf{4 1})\end{array}$ & $\begin{array}{c}\text { Unimpaired } \\
(\mathbf{n}=\mathbf{9 4})\end{array}$ & $\boldsymbol{P}$ \\
\hline Age, years & $58.6 \pm 10.8(25-87)$ & $59.5 \pm 11.2(31-82)$ & $58.2 \pm 10.6(25-87)$ & 0.544 \\
Women & $73(63.5)$ & $22(61.1)$ & $51(64.6)$ & 0.883 \\
White & $93(80.9)$ & $25(69.4)$ & $68(86.1)$ & 0.065 \\
Education, <12 years & $18(15.7)$ & $10(27.8)$ & $8(10.1)$ & $0.032^{\dagger}$ \\
Married/with partner & $72(62.6)$ & $20(55.6)$ & $52(65.8)$ & 0.350 \\
CVD risk factors & $2.1 \pm 1.7(0-6)$ & $2.7 \pm 1.5(0-6)$ & $1.8 \pm 1.7(0-6)$ & $0.003^{\dagger}$ \\
Income, <\$20,000 & $9(7.8)$ & $6(16.7)$ & $3(3.8)$ & $0.045^{\dagger}$ \\
Oral glucocorticoid use & $39(33.9)$ & $15(41.7)$ & $24(30.4)$ & 0.330 \\
Duration of RA, years & $19.6 \pm 11.3(0-56)$ & $17.6 \pm 9.3(4-37)$ & $20.6 \pm 12.1(0-56)$ & 0.202 \\
Severity of RA & $2.4 \pm 1.6(0-6.7)$ & $2.6 \pm 1.8(0-6.1)$ & $2.2 \pm 1.5(0-6.7)$ & 0.291 \\
C-reactive protein level $(>3 \mathrm{mg} / \mathrm{dl})$ & $41(35.7)$ & $15(41.7)$ & $26(32.9)$ & 0.484 \\
Depression & $8(7.0)$ & $5(13.9)$ & $3(3.8)$ & 0.115 \\
\hline
\end{tabular}

Values are the mean \pm SD (range) or no. $(\%)$.

$\mathrm{CVD}=$ cardiovascular disease RA = rheumatoid arthritis.

${ }^{\dagger}$ Significant at $P<0.05$. 
Table 2

Logistic regression analysis: predictors of cognitive impairment ${ }^{*}$

\begin{tabular}{|lll|}
\hline & \multicolumn{2}{c|}{ OR $(\mathbf{9 5 \%}$ CI $)$} \\
\cline { 2 - 3 } Predictors & Bivariate & Multivariate \\
\hline Women (men vs. women) & $0.86(0.38-1.95)$ & $1.30(0.48-3.55)$ \\
Race (white vs. nonwhite) & $2.72(1.05-7.06)^{\dagger}$ & $3.00(0.85-10.56)$ \\
Education $(>12$ years vs. $<12$ years) & $3.41(1.22-9.59)^{\dagger}$ & $6.18(1.60-23.87)^{\dagger}$ \\
Income $(>\$ 20 \mathrm{k}$ vs. $\langle \$ 20 \mathrm{k})$ & $5.07(1.19-21.58)^{\dagger}$ & $7.12(1.35-37.51)^{\dagger}$ \\
Depression & $4.09(0.92-18.15)$ & $2.65(0.44-16.17)$ \\
Severity of RA & $1.15(0.90-1.47)$ & $0.83(0.59-1.16)$ \\
Duration of RA & $0.98(0.94-1.01)$ & $0.96(0.92-1.01)$ \\
C-reactive protein level $(<3$ vs. $>3 \mathrm{mg} / \mathrm{dl})$ & $1.46(0.65-3.28)$ & $1.21(0.43-3.38)$ \\
Oral steroid use & $1.64(0.72-3.71)$ & $2.92(1.05-8.12)^{\dagger}$ \\
CVD risk factor score & $1.39(1.08-1.77)^{\dagger}$ & $1.61(1.19-2.17)^{\dagger}$ \\
\hline
\end{tabular}

*ardiovascular disease risk factor score: hypertension, high blood pressure medications, systolic blood pressure, total cholesterol, high-density lipoprotein cholesterol, obesity, and smoking status.

$\mathrm{OR}=$ odds ratio; $95 \% \mathrm{CI}=95 \%$ confidence interval; $\mathrm{RA}=$ rheumatoid arthritis $\mathrm{CVD}=$ cardiovascular disease.

${ }^{\dagger}$ Significant at $P<0.05$. 